EXEMPLARIa Classica

Journal of Classical Philology

23, 2019, 243-252 • ISSN 1699-3225

http://dx.doi.org/10.33776/ec.v23i0.3781

\title{
SUCCESSIVI SVILUPPI DI THE MANUSCRIPT TRANSMISSION OF THE ANTHOLOGIA LATINA*
}

\author{
Paola Paolucci \\ Università degli Studi di Perugia \\ paola.paolucci@unipg.it
}

Il volume di Loriano Zurli citato nel titolo è stato pubblicato nella Collana Anthologiarum Latinarum Parerga per i tipi di Olms (Weidmann) nel 2017 e costituisce traduzione inglese (letteralissima e litteratissima), a cura di Vincenzo Russo e Paola Tempone, del volume del medesimo Loriano Zurli, La tradizione ms. di Anthologia Latina, pubblicato a Perugia nel 2014 (Editrice Pliniana; ISBN 978-88-97830-30-6) nell'ambito delle Pubblicazioni del Centro Studi Anthologia Latina, già positivamente recensito da Marco Giovini (in Res Publica Litterarum n.s. 17, 2014, 222-9). A sua volta, questo volume del 2014, ampliava ed aggiornava un lungo articolo sul medesimo argomento, comparso nel primo numero di " $A L$. Rivista di Studi di Anthologia Latina" (2010). E nel detto lungo articolo confluivano le ricerche, tutte convergenti verso un'organica delineazione dello stemma codicum di Anthologia Latina, pubblicate alla spicciolata in vari articoli e nelle prefazioni a più di un'edizione critica nel corso di oltre vent'anni di studi. Come si vede, dunque, l'argomento della tradizione ms. delle due più cospicue anthologiae (la Salmasiana e la Vossiana) della raccolta comunemente denominata Anthologia Latina ha fatto registrare via via progressi ed esigenze di divulgazione sempre crescenti che non si sono di certo arrestati nel biennio (scarso) intercorso fra la data di pubblicazione del volume in inglese (2017) ed il momento odierno, né deo favente si interromperranno in futuro, essendo già chiare nuove ed ulteriori piste di indagine ed essendo fertile (in modo direttamente proporzionale all'iniquitas et malignitas temporum) il vivaio della Scuola perugina di Filologia latina che se ne occupa.

Perciò mi propongo di indicare qui, dopo un sommario riepilogo del piano dell'opera a pro del lettore ignaro, le novità più recenti susseguenti agli sviluppi di questa stessa opera e di abbozzare nuove proposte di indagine ed ampliamenti futuri.

Richiamo nel titolo del mio contributo il titolo del volume di L. Zurli, The Manuscript Transmission of the Anthologia Latina, Hildesheim 2017, dal quale muove il presente contributo. 
Il lettore di The Manuscript Transmission of the Anthologia Latina è condotto gradualmente all'illustrazione finale dello stemma codicum delle antologie Salmasiana e Vossiana attraverso un percorso a tappe costituito da capitoli inerenti i seguenti soggetti, che vengono investigati a tutto tondo: il codex Parisinus Latinus 10318 (Salmasianus; A); il codex Parisinus Latinus $8071^{1}$ o Thuaneus; il carme $A L 389 \mathrm{R}^{2}$, definibile anch'esso come Salmasiano, benché assente nel codice così denominato; il rapporto fra il codex Parisinus Latinus 8071, il Vossianus Latinus Q. 86 e gli excerpta Martialis; il codex Vindobonensis Palatinus 9401*, cui ségue una rideterminazione sul c.d. ramo $\beta$ della tradizione, rappresentato dai testimoni non-salmasiani di Anthologia Latina (d'ora in poi $A L$ ); il codice Vossiano $(Q .86)$ e la costituzione del corpus degli epigrammi attribuiti a Seneca; gli altri codici che trasmettono gruppi di carmi o singoli componimenti di $A L$, come il deperditus codex Bellovacensis del Binet, il Parisinus Latinus 8069, il Reginensis Latinus 123, il Lipsiensis Rep. I 74, il Parisinus Latinus 9344, il Berolinensis Diez. B Sant. 66, il Casanatensis 641, il Sangallensis 899, il Regius mus. Brit. 15 B 19, il Petropolitanus F 14, 1 e il Laurentianus plut. 33,31; la tradizione umanistica; gli errori di archetipo e gli stemmi di $A L$ già prodotti; per finire, appunto, con la proposta di un nuovo stemma di $A L$. In Appendice figurano alcune novità desumibili dall'epistolario Is. Voss - Cl. Saumaise.

Sul tema del capitolo iniziale, dedicato al codice Salmasiano, lo scorso anno (2018), sempre per i tipi dell'Editrice Pliniana, sono stati pubblicati in volume dal titolo Il codice Salmasiano (Par. Lat. 10318). I suoi testi, le sue immagini (cur. L. Zurli), gli atti del Seminario internazionale, tenutosi a Perugia il 15 marzo 2018. Gli elementi di novità emersi in quel contesto sono stati vari, ma soprattutto si sono segnalati i seguenti apporti: una definitiva condivisione del fatto che a monte di A si situi un ms. in scrittura semionciale, una ridefinizione della paleografia del codice ed in particolare l'individuazione della provenienza amiatina dello stesso codice (così Zurli in Schegge Salmasiane, in Appendice) e la definizione delle tappe della sua circolazione medioevale. Si è a questo fine approfondita l'indagine (da parte di Tino Licht) del rapporto intercorrente fra A ed il codice Vaticano Reginense 123 (sec. XI, Monastero di Ripoll), che secondo Riese avrebbe derivato da A i due carmi da esso condivisi con questo testimone ms. Il che tuttavia non vuol significare che la biblioteca d'origine del Salmasiano sarebbe stata quella di Ripoll, ma che, considerati i numerosi scambi fra Ripoll e Fleury nel sec. XI, sede di conservazione del Salmasiano possa essere stata appunto la Biblioteca di Fleury.

${ }^{1}$ Cf. D. Antiseri, Teoria unificata del metodo, Torino 2001.

${ }^{2}$ Cf. M. Spallone, "Il Par. Lat. 10318 (Salmasiano): dal manoscritto alto-medievale ad una raccolta enciclopedica tardo-antica", IMU 25, 1982, 1-71. 
Considerato che Tino Licht ha preliminarmente esaminato e confermato i collegamenti formali fra il codice Salmasiano e noti testimoni mss. confezionati nello scriptorium Amiatino o ivi posseduti, si può concludere che esso, realizzato al Monte Amiata, presto sarebbe migrato a Fleury, dove sarebbe rimasto fino all'età delle guerre di religione in Francia e precisamente fino al salvataggio della biblioteca di Fleury ad opera di Pierre Daniel nel 1562. E ciò collima (se non perfettamente, di certo con considerevole approssimazione geografica e temporale) con le conclusioni alle quali ero pervenuta (in "Hosidiana", Paideia 65, 2010, 555-72) attraverso l'esame della corrispondenza epistolare di dotti umanisti del sec. XVI intorno alla Medea di Osidio/Ovidio; scrivevo, infatti: "Il baillage di Arnai... fu teatro d'importanti scontri durante le guerre di religione e dopo la battaglia fra l'ammiraglio di Coligny e il maresciallo di Cossé-Brissac, il 27 giugno 1570, risoltasi con successo per i Protestanti, Ugonotti e Calvinisti vi trovarono una sede sicura. Perciò il codice Salmasiano potrebbe essere giunto ad Arnai con qualche famiglia aristocratica ugonotta/calvinista che vi aveva trovato rifugio (per questa ragione risultano curiose le dicerie circa la presenza della Medea di Ovidio/Osidio presso il Pucheimer, che si muoveva nella cerchia dei Fugger, di Erasmo e di Peutinger, tutti insigni personaggi che ebbero ruoli di spicco anche nelle controversie religiose del periodo della Riforma); a meno che... durante le guerre di religione, esso non sia stato trafugato da uno dei numerosi monasteri della zona (ci si trova nella diocesi di Autun e non è distante il celeberrimo Cluny), dove era custodito da tempo» (555-6).

Ad ogni buon conto, in merito all'individuazione dello scriptorium di origine del codice Salmasiano, non fosse che - come ci hanno insegnato due padri illustri della teoria della scienza e della epistemologia, come Popper e Gadamer $^{1}$ - a fini di falsificazione e dunque di prova della tenuta dell'ipotesi amiatina, mi permetto di suggerire anche un'altra pista di indagine. Riterrei, infatti, utile che si tentasse un raffronto fra il manufatto Salmasiano e la produzione dell'abbazia di Farfa, il cui nucleo originario pare risalga al VI sec. Orbene, se - come dicevo - le indagini da me svolte sulla probabile collocazione del Salmasiano prima della sua riscoperta nel $1615 \mathrm{mi}$ portavano ad individuare l'area di Autun e di Cluny, le indagini da me condotte sulla grafia (per me semionciale) dell'antigrafo del Salmasiano mi portavano a corroborare l'ipotesi, affacciata già da Maddalena Spallone ${ }^{2}$, che «la silloge cartaginese», tramandata nel codice Salmasiano, abbia «seguíto, una volta dipartitasi dall'Africa, lo stesso percorso verso il castrum Lucullanum di Eugippio effettuato da altri testi di provenienza africana e vergati/copiati in loco (cioè nel monastero napoletano di San Severino, nella metà del VI sec.) prevalentemente nella scrittura ivi prediletta: la semionciale» ${ }^{3}$. Occorrerebbe,

${ }^{3}$ Cf. P. Paolucci, "Quale grafia a monte del codice Salmasiano?", ALRiv 1, 2010, 295. Sull'ipotesi di una migrazione dell'Anthologia Salmasiana dall'Africa alla Spagna visigotica 
dunque, se questi due presupposti hanno fondamento, provare a colmare il gap (non irrilevante) che si frappone fra i due termini spazio-temporali sopra ricordati (intendo la regione Campana nella seconda metà del VI sec. e l'area cluniacense nella seconda metà del XVI sec.) e crederei che in questo esteso gap un ruolo assai importante possa essere stato svolto dall'abbazia di Farfa.

È noto che dopo la prima fondazione nel VI sec., intorno all'anno $680 \mathrm{si}$ ebbe la rifondazione del monastero ad opera di Tommaso da Moriana. Come si apprende dalla Constructio Monasterii Farfensis, sin da quella data il nuovo sito abbaziale divenne polo di attrazione, al punto che ceperunt ad eum tam de proximis quam de longinquis provinciis certatim concurrere (cf. Lectio VI della citata Constructio) ed in particolare si intrecciarono a doppio filo i rapporti tra Farfa e Benevento, stanti le lectiones VI e XI della medesima Constructio, che raccontano il pellegrinaggio di tre nobili Beneventani a Tommaso e di Tommaso a Benevento con successiva fondazione del monastero di san Vincenzo al Volturno: Eodem igitur tempore fuerunt Benevento tres nobili exorti progenie viri... Erat enim eodem tempore Gisulfus vir spectabilis, Beneventani ducatus tenens principatus e.q.s. La prima notizia di un'intensa attività di copiatura di codici a Farfa risale agli anni 761-769, corrispondenti al periodo di reggenza del sesto abate della congregazione farfense, cioè Alano. Di lui la Constructio afferma: Sextus denique in hac congregatione Alanus extitit pater, regione exortus Aquitania... qui tam spiritalis philosophie quam etiam secularis astutie prudentissimus fuit... in Motilla quoque monte, qui hoc supereminet monasterium... per annos deguit multos inclausus; ubi inter alia bone operationis exercitia multos etiam mirifice exaravit codices. La notizia è confermata e ribadita dal Chronicon Farfense di Gregorio da Catino (10621133). Un periodo di particolare opulenza visse l'abbazia di Farfa negli anni 790-802 e 802-815 (si ricordi che il Salmasiano è datato all'anno 800 ca.) ${ }^{4}$, cioè sotto il decimo e l'undicesimo abate, dei quali la Constructio riferisce: Decimus quoque huic monasterio in sancto prefuit regimine vir vite venerabilis Mauroaldus, natione Francus... nam in ecclesia beate Marie

cf. B. Bischoff, Manuscripts and Libraries in the Age of Charlemagne, transl. and edited by M. Gorman, Cambridge 1994, 3. Rimangono tuttavia celebri, a proposito del considerevole flusso di codici lungo la direttrice Africa settentrionale-Italia meridionale, le due testimonianze di Cassiod. Inst. 1.8.9 qui vobis inter alios codices divina gratia suffragante de Africana parte mittendus est e 1.29.2 cuius dicta Victor Mattaritanus, episcopus Afer, ita Domino iuvante purgavit, et quae minus erant addidit, ut ei rerum istarum palma merito conferatur; quem inter alios de Africae partibus cito nobis credimus esse dirigendum. Mi preme, inoltre, aggiungere che sin da epoca remota si instaura un rapporto molto stretto fra il popolo dei Longobardi (che poi scenderanno in Italia e contribuiranno alla fondazione di San Salvatore sull'Amiata - Ratchis - o tesseranno relazioni con importanti abbazie dell'Italia centro-meridionale, tra cui Farfa) e San Severino, cioè il santo al quale è dedicato il monastero al Castrum Lucullanum presso Napoli. Lo testimonia espressamente Paul. Diac. Hist. Lang. 1.19.

${ }^{4}$ Cf. Bischoff, Manuscripts, 44. 
multum pretiosum ex auro et argento ornatum fieri iussit... Undecimus denique huic monasterio prefuit pater vir venerabilis Benedictus, qui in eis que ad Deum pertinent valde extitit devotus ad suam fratrumque salutem, necnon et in exterioribus ornamentis que ad cultum ecclesie utilia videntur; ita ut in libris... nimis sagacissimus esset. Il collegamento del monastero con Benevento portò con sé il coinvolgimento di importanti aristocratici longobardi che furono anche duchi a Spoleto e che affiorano passim in numerosi luoghi del Chronicon Farfense, assai più generoso di dettagli che non la Constructio. Basta seguire con l'occhio i Catalogi premessi al Chronicon di Greogorio da Catino per trovare questi duchi elencati in bell'ordine nei termini seguenti:

\section{Transmundus dux Spoleti comes dudum Campanus}

Faroaldus filius suprascripti Transmundi dux Spoleti

Transmundus contra patrem suum Faroaldum rebellans, clericum illum fecit et ducatum Spoletanum invasit

Hildericus dux Spoleti.

Come si vede, questi signorotti longobardi, che si muovono fra la Campania e Spoleto ed intessono fitte relazioni con Farfa, si chiamano Trasamondo (nonno e nipote) ed Ilderico, cioè sono omonimi dei più insigni sovrani vandalici cantati dai maggiori poeti della silloge Salmasiana, che in quegli anni doveva trovarsi in Campania (se le mie ricerche sulla semionciale a monte del Salmasiano sono, come è parso a più d'uno, fondate). Sarà questo un caso? Probabilmente no, potendosi verisimilmente credere che la silloge cartaginese possa aver vissuto in questo ambiente un suo Revival proprio in virtù di certa ominimia oppure sia stata proprio quella raccolta poetica ad ispirare la denominazione di quei duchi. Una nota conseguenza di tale curiosa coincidenza nominale fu che Bernardino Corio fra il 1485 e il 1502 confuse il Trasamondo elogiato da Draconzio con il Trasamondo, principe di Capua $^{5}$, che poi divenne duca di Spoleto, come ci riferisce Paolo Diacono ${ }^{6}$. ̀̀

${ }^{5}$ Cf. P. Paolucci, "H. Kaufmann, Dracontius, Romul. 10 (Medea): Einleitung, Text, Übersetzung und Kommentar, Hiedelberg: Winter, 2006, 558 pp., ISBN 3-8253-5142-4", ExClass 11, 2007, 502-3.

${ }^{6}$ Paolo Diacono, dopo aver parlato di Faroaldo, primo duca di Spoleto (Hist. Lang. 3.13), narra le imprese di Trasamondo, conte di Capua, ordinate da Grimoaldo, e la sua nomina a duca di Spoleto (cf. Hist. Lang. 4.51 Transemundum vero comitem Capuanum per Spoletium et Tusciam direxit, ut eius regionis Langobardos suo consortio coaptaret. Qui imperata sibi strenue perficiens, ei cum multis adiutoribus aput Aemiliam in itinere occurrit; 5.16 At vero rex Grimuald, ereptis Beneventanis et eorum provinciis a Graecis, ad palatium suum aput Ticinum repedare disponens, Transamundum, qui dudum Capuae comes 
un fatto, in ogni caso, che Trasamondo ed Ilderico, che peraltro non possono considerarsi idionimi propriamente longobardi ${ }^{7}$, si trovano in successione nel ducato di Spoleto come un tempo (496-530) i loro omonimi a Cartagine. Ed altro fatto è che uno dei testimoni ms. minori, seriori e parziali dei carmi di Anthologia Salmasiana, il Lipsiensis I $74^{8}$, contiene anche l'epitafio della regina longobarda Ansa, attribuito a Paolo Diacono.

Vero è che la diaspora dei mss. del monastero di San Severino raggiunse mete plurime: ad es., il Sessorianus 13, datato al VI sec., con note di Eugippio, costituente il più antico testimone del De genesi ad litteram di Sant'Agostino (= Biblioteca Nazionale di Roma 2094), avrebbe raggiunto l'abbazia di Nonantola. Tuttavia va detto che il cod. Farf. 29 (= Biblioteca Nazionale di Roma 341), di IX sec. (842-898), uno dei mss. vetusti di Farfa che passarono alla Biblioteca Nazionale di Roma, lascerebbe presumere vista l'onciale delle sue rubriche - che nel sec. IX nello scriptorium Farfense operasse almeno uno scriba educato all'uso dell'onciale romana, ovvero di quella tipizzazione dell'onciale che Maddalena Spallone ${ }^{9}$ ha individuato come propria dello scriba del Salmasiano ${ }^{10}$.

Nell'anno 898 Farfa subì l'invasione Saracena, ma grazie all'abate Ugo (997-1038) fu in grado di rifiorire e di ospitare Sant'Odilone di Cluny, di cui adottò l'osservanza. E ciò salderebbe le vicende italiane della silloge cartaginese con l'area gallica della riemersione del Salmasiano.

Qualunque sia stato lo scriptorium dell'Italia centrale (questo sì, pare assodato), nel quale fu realizzato il Salmasiano, si dovrà considerare come fatto

fuerat et ei ad percipiendum regnum strenuissime paruerat, data ei in matrimonium sua filia, Romualdi altera sorore, eum post Attonem, de quo superius diximus, aput Spoletium ductorem effecit, indeque Ticinum reversus est). Trasamondo, duca di Spoleto, morì nel 703 e gli successe il figlio Faroaldo, che nel 716 fu deposto dal figlio Trasamondo (Hist. Lang. 6.30 e 6.44). Costui si ribellò a Liutprando, ma fu costretto a rifugiarsi a Roma, lasciando il suo posto ad Ilderico che fu ucciso successivamente dal medesimo Trasamondo, rientrato da Roma, che fu definitivamente deposto da Liutprando (Hist. Lang. 6.55 e 57).

7 Tipici idionimi longobardi popolano le pagine della Historia Langobardorum di Paolo Diacono. Tra questi possiamo ricordare, ad es., Cunicperto, Ansprando, Liutprando, Ratchis, Arichis. Sull'onomastica di questi popoli cf. N. Francovich Onesti, I Vandali. Lingua e storia, Roma 2002 e Ead., I nomi degli Ostrogoti, Firenze 2007.

${ }^{8}$ Questo codice contiene i carmi $A L 389 \mathrm{R}^{2}$ al f. 14; 160, 39, 97, 23, 276, 261, 259, 256, 257 $\mathrm{R}^{2}$ ai ff. 25-27.

${ }^{9}$ Cf. Spallone, "Il Par. Lat.", 47.

${ }^{10} \mathrm{Cf}$. A. Petrucci, "L'onciale romana. Origini, sviluppo e diffusione di una stilizzazione grafica altomedievale (sec. VI-IX)", Studi Medievali, s. 3, 12, 1971, 75-132; P. Supino Martini, "Carolina romana e minuscola romanesca. Appunti per una storia della scrittura latina in Roma tra IX e XII secolo", Studi Medievali, s. 3, 15, 1974, 769-93; Ead., "La produzione libraria negli scriptoria delle abbazie di Farfa e sant'Eutizio", Atti del IX Congresso internazionale di studi sull'Altomedioevo, Spoleto, 27 settembre-2 ottobre 1982, vol. 2, 1983, 581-607; Ead., Roma e l'area grafica romanesca, Alessandria 1982; S. Nocentini, Le passioni di San Miniato martire fiorentino, Firenze 2018, 75-6; G. Brugnoli, "Catalogus codicum Farfensium”, Benedictina 7, 1953, 104-11. 
altamente probabile la circostanza che forte impulso alla circolazione dei carmi della silloge cartaginese, approdati in Italia e trascritti in $\alpha$, abbiano dato $i$ signori Longobardi ed in particolare Trasamondo conte di Capua, duca di Spoleto, ed i suoi successori che si scontrarono a più riprese con Liutprando. Lo testimonia, a mio modesto avviso, l'evidente motto iniziale al verso incipitario di un carme tràdito soltanto da $\mathrm{A}$, cioè ad $A L 20 \mathrm{R}^{2}=7 \mathrm{SB}$ Versus Octaviani viri inlustris annorum XVI filius Crescentini viri magnifici, v. 1 Candida sidereo fulgebat marmore Cypris, nel primo esametro (anch'esso aureo) dell'epitafio del vescovo Pietro di Pavia, congiunto del re Liutprando, morto nel 736: Candida funereo sculpuntur marmore gesta11.

Le conoscenze in merito ai testimoni mss. di Anthologia Latina hanno potuto beneficiare negli ultimi anni anche degli apporti di recenti edizioni critiche di singoli carmi o sezioni della medesima silloge. Tacerò delle mie edizioni dei centoni Salmasiani Hippodamia ed Alcesta, pubblicate per i tipi di Olms rispettivamente nel 2006 e nel 2015, ma non posso esimermi dal riferire un apporto importante, scaturito dallo studio di due corruttele di Alcesta, alla delineazione della fisionomia del glossatore di $\alpha$, cioè dell'antigrafo del Salmasiano ${ }^{12}$, utile a spiegare sotto una nuova luce vari luoghi corrotti di Anthologia Salmasiana ed in particolare il problema della fase alta della tradizione ms. degli Aenigmata Simposii ${ }^{13}$, come sagacemente ha saputo cogliere Giulio Vannini nella sua recente edizione dell'Historia Apollonii regis Tyri $^{14}$.

Contributo importante alla storia della tradizione ms. dei Carmina XII sapientum ha fornito di recente l'accuratissima edizione critica di questi carmi, con traduzione poetica e commento filologico, di Luca Martorellii' comparsa nella Collana Anthologiarum Latinarum Parerga, diretta da L. Zurli presso Olms. La pregevole introduzione fornisce informazioni complete ed esatte (senza omettere la sitografia utile al reperimento delle riproduzioni digitali dei codici) sui numerosissimi testimoni mss. dell'interessante raccoltina di cicli epigrammatici ascritta ai dodici sapientes, edita a suo

${ }^{11}$ Questo epitafio, che Dümmler pubblica fra i Tituli saeculi VIII, del I tomo dei Poetae aevi Carolini, è testimoniato nel codex Vaticanus Palatinus 833 (sec. IX-X). Sul vescovo Pietro cf. Paul. Diac. Hist. Lang. 6.58.

${ }^{12}$ Cf. P. Paolucci, "Dall'Alcesta centonaria ad alcune chiose di lettura nella tradizione a monte del Salmasiano", in P. Mastandrea - L. Spinazzè (edd.), Nuovi archivi e mezzi d'analisi per $i$ testi poetici, Amsterdam 2011, 239-49; Ead., Studi sull'Alcesta centonaria, Perugia 2014, 67-77; Ead., Il centone virgiliano Alcesta dell'Anthologia Latina, Hildesheim 2015, CCI sg.; Ead., "Per la costituzione del testo dell'Alcesta centonaria. L'epilogo", in M.T. Galli G. Moretti (edd.), Sparsa colligere et integrare lacerata. Centoni, pastiches e la tradizione greco-latina del reimpiego testuale, Trento 2014, 57-77.

${ }^{13}$ Cf. P. Paolucci, "La mela dell'inganno" ALRiv 5, 2014, 105-12.

${ }^{14}$ Cf. G. Vannini, Storia di Apollonio re di Tiro, Milano 2018.

${ }^{15} \mathrm{Cf}$. L. Martorelli, Versus sapientum de diversis causis, Hildesheim 2018. 
tempo (1906) da Riese ${ }^{16}$ nel II tomo di Anthologia Latina e non del tutto convincentemente ripubblicata dalla Friedrich nel 2002 ${ }^{17}$.

Nel capitolo dedicato al codice Vossiano Q. 86 e agli epigrammi di Seneca (The Manuscript Transmission, 120-8) Zurli sostiene, molto opportunamente, che «aiming at the knowledge of the evolutionary lines of the transmission of the 'Senecan' texts, as well as of other texts which are connected to them in $\mathbf{V}$, the study of the indirect transmission and of the Fortleben in authors of the Middle Ages - those till now 'found out' are all Carolingian, as well as Voss. Q. 86 - is an external possibility of progress one should not miss». Effettivamente questo suggerimento non deve essere negletto, perché è foriero di interessanti acquisizioni, come ho potuto verificare a proposito del carme De habitatione ruris $\left(A L 26 \mathrm{R}^{2}\right)$, noto a Rabano Mauro tramite V, in un mio intervento dal titolo "Gargilio Marziale e Valafrido Strabone. Sulle tracce di un rapporto intertestuale", in corso di stampa negli atti del Convegno straboniano tenutosi ad Heidelberg il 27-29 settembre 2018, e come mi appresto a mostrare qui con almeno un nuovo argomento, reperito spigolando fra i Poetae aevi Carolini di $M G H$.

Il primo poeta di questa collezione è - come è noto - Bonifatius che è attivo nel primo quarto del sec. VIII, cioè grosso modo all'altezza cronologica della posizione stemmatica di $\beta \gamma$ da cui BV discendono. Egli, esperto nell'arte grammatica, nell'eloquenza e nella versificazione (come ci insegna la Vita $S$. Bonifatii 2) è celebre per la sua produzione di enigmi acrostici, in continuità con quella di Sinfosio ${ }^{18}$ (la cui raccolta convive con quella di Bonifatius nel codex Petropolitanus, quondam Corbeiensis 598, S. Germani Prat. 783 , sec. VIII-IX), dedicata ad una soror, non necessariamente germana, di nome Leobytha o Lioba. Di lui c'interessa il prologo agli enigmi, che è testimoniato, oltre che dal complesso dei mss. trasmettenti la raccolta, anche dal f. 86 del codex bibliothecae Universitatis Turicensis C. 78.451 (sec. IX-X), originario di San Gallo. Gli enigmi fin dall'esordio vi sono assimilati a mele d'oro:

I.

INCIPIUNT AENIGMATA BONIFATII EPISCOPI QUAE MISIT SORORI SUAE

Aurea nam decem transmisi poma sorori, Quae in ligno vitae crescebant floribus almis,

${ }^{16}$ Cf. A. Riese, Anthologia Latina, t. II, Lipsiae $1906{ }^{2}$.

${ }^{17} \mathrm{Cf}$. A. Friedrich, Das Symposium der XII Sapientes. Kommentar und Verfasserfrage, Berlin-New York 2002.

${ }^{18}$ Cf. G. Polara, "Aenigmata”, in G. Cavallo, C. Leonardi, E. Menestò (edd.), Lo spazio letterario del Medioevo, vol. I, t. II, Roma 1993, 210-2. 
Illius et sacris pendebant dulcia ramis, Cum lignum vitae pendebat in arbore mortis.

Cum quibus et ludens conprendas gaudia mentis

Et tibi venturae conplearis dulcedine vitae,

Manducans mulso inspireris nectaris haustu.

Spirantes replet nardi flagrantia nares.

Cum quibus et malis conpares regna futura:

10 Dulcia sic quondam celebrabis gaudia caeli.

Sunt alia alterius ligni acerbissima mala,

Pestifero vernant quae in ligno mortis amarae,

Quae Adam manducans dira est cum morte peremptus,

Antiqui infecta et flatu et felleque draconis,

15 Vipereo ut dudum saeve perlita veneno.

Nitatur palmis haec numquam tangere virgo,

Mandere quae nefas est et gustare profanum,

Ne dentes strideant fuscati peste maligna,

Talibus aut malis frangantur foedera sancta,

20 Vel superi incassum perdantur premia regni.

Come giustamente riporta il secondo apparato (n. 2) ${ }^{19}$ di Dümmler, il primo verso echeggia Verg. ecl. 3.71 aurea mala decem misi..., su Menalca che dona pomi al puer amato, ma con la cautela che pur richiedono argomenti di tal genere, crederei non debba esser taciuto il modello dell'epigramma ps.petroniano $A L 218 \mathrm{R}^{2}=209 \mathrm{SB}$, presente in ABVS, intitolato De malis aureis amatori ab amata missis (ma De malis aureis V), concernente lo scambio del dono di mele d'oro fra una donna ed un uomo ${ }^{20}$ (a parti invertite, dunque, rispetto al carme medioevale), il cui tono erotico non sarebbe assolutamente ostativo al suo echeggiamento nell'incipit degli esametri moralizzati (v. 5 gaudia mentis) di Bonifatius. Eccone il testo nell'edizione di Shackleton Bailey:

Aurea mala mihi, dulcis mea Martia, mittis, mittis et hirsutae munera castaneae.

Omnia grata puta; sed si magis ipsa venires, ornares donum, pulchra puella, tuum.

Tu licet adportes stringentia fella palatum, tristia mandenti est melleus ore sapor.

At si dissimulas, multum mihi cara, venire, oscula cum pomis mitte: vorabo libens.

${ }^{19} \mathrm{Si}$ sarebbe potuto citare anche Auson. Epist. 16.1.

${ }^{20}$ Cf. R.M. D'Angelo, “AL $218 \mathrm{R}^{2}=209$ SB fra simbolismo e mixtio generum”, in L. Zurli (ed.), Il codice Salmasiano (Par. Lat. 10318). I suoi testi, le sue immagini, "Atti del Seminario internazionale, Perugia, 15 marzo 2018”, Perugia 2018, 53-76. 
Come si può notare, le sfide alle quali ci chiama l'Anthologia Latina sono numerose ed avvincenti e molti sono ancora i campi nei quali ci possiamo cimentare, forti delle conoscenze che ci vengono impartite da The Manuscript Transmission of the Anthologia Latina. 